\title{
Profil des métaux lourds contenus dans les plantes vivrières consommées couramment dans quelques zones minières de la province du Katanga.
}

\author{
Dominique Mudimbi Kalonda ${ }^{1}$, Arsène Kabamba Tshikongo', Fridolin Kodondi Kule koto², Christian \\ Kasongo Busambwa ${ }^{1}$, Yves Kisunka Bwalya1', Hervé Musola Cansa ${ }^{1}$, Jean-Louis Kahambwe \\ Tambwe ${ }^{1}$, Zet Lukumwena Kalala ${ }^{3}$, Albert Longanga Otshudi 1,4 \\ (1) Faculté des Sciences pharmaceutiques, Université de Lubumbashi, RD Congo \\ (2) Faculté des Sciences pharmaceutiques, Université de Kinshasa, RD Congo \\ (3) Faculté de Médecine Vétérinaire, Université de Lubumbashi, RD Congo \\ (4) Université Libre de Bruxelles, Belgique. \\ Auteur correspondant Pharmacien Arsène KABAMBA, Faculté des Sciences Pharmaceutiques, Université de \\ Lubumbashi, République Démocratique du Congo. arsene.kabamba@gmail.com; arsenekabamba@yahoo.fr. Tél. +243 \\ 812143587 ; +243992143587.
}

Original submitted in on 19th October 2015. Published online at www.m.elewa.org on 31st December 2015 http://dx.doi.org/10.4314/jab.v96i1.2

\section{RESUME}

Contexte : Le transfert des éléments polluants, et notamment des métaux lourds fait partie des problèmes graves de santé publique. La contamination des plantes vivrières par les polluants, et notamment les métaux lourds, est une préoccupation sociétale majeure. En effet, de plus en plus de cultures sont implantées en milieu périurbain, et de ce fait peuvent être impactées par des industries polluantes passées ou encore en activité. Cette problématique est à l'échelle mondiale, et très peu de pays sont épargnés. Ces contaminations peuvent être liées à une pollution des sols, ou des eaux utilisées en irrigation.

Objectif: L'objectif de ce travail était de déterminer le profil des métaux contenus dans Manihot esculenta (Sombe); Amaranthus (Lengalenga) et Psidium guajava L. (Mapela) ; plantes vivrières utilisées en alimentation dans les zones aux alentours des sites miniers dans la province du Katanga, en République Démocratique du Congo.

Méthodes et Résultats : II était question dans ce travail d'une étude prospective analysant les feuilles des plantes précitées. Plantes collectées sur le site maraicher de quelques zones minières dans la province du Katanga. La recherche des métaux lourds dans les échantillons a été réalisée par la spectroscopie de masse (ICP-MS). Les analyses ont révélés la présence des métaux lourds dans les échantillons, et cela à des concentrations différentes. Toutefois les taux de zinc, cuivre, cadmium, plomb et autres métaux lourds étaient au-delà des limites normales.

Conclusion et application des résultats : Les plantes Manihot esculenta (Sombe); Amaranthus (Lengalenga); et Psidium guajava L. (Mapela) se trouvant aux alentours des zones minières de la province du Katanga, renferment les métaux lourds. Dans la plupart des cas, ces métaux sont au delà des seuils normaux. Ce qui pourrait être à la base des cas d'intoxication. Ces résultats permettront la mise en route des mesures préventives par rapport à la culture vivrière dans ces zones. 


\section{Kalonda et al. J. Appl. Biosci. 2015 Profil des métaux lourds contenus dans les plantes vivrières}

consommées couramment dans quelques zones minières de la province du Katanga.

Mots Clés : Métaux lourds, Mines, Katanga.

\section{ABSTRACT}

Context: The contamination of food crops by heavy metals is one major public health problem. Indeed, more and more crops are located in suburban areas, and thus can be affected by past or active polluting industries. This problem is global, and few countries are spared. These contaminations can be related to soil pollution, or water used for irrigation.

Objective : The objective of this work was to determine the profile of the metals contained in Manihot esculenta (Sombe); Amaranthus (Lengalenga) and Psidium guajava L. (Mapela), plants used in areas around the mining sites in Katanga Province, Democratic Republic of Congo.

Methodology and Results: At issue in this work of a prospective study analyzing the leaves of the aforementioned plants. Plants collected on the site maraicher some mining areas in Katanga province. The presence of heavy metals in the samples was performed by mass spectroscopy (ICP-MS).The analyzes revealed the presence of heavy metals in the samples, and this in different concentrations. However the levels of zinc, copper, cadmium, lead and other heavy metals were beyond normal limits.

Conclusion and application of results: Plants Manihot esculenta (Sombe) ; Amaranthus (Lengalenga) and Psidium guajava L. (Mapela), located around the mining areas of Katanga province, contain heavy metals. In most cases, these metals are beyond the normal limits. What could be the basis of poisoning. These results will allow the initiation of preventive measures in relation to food crops in these areas.

Keywords: Heavy metals, Mining, Katanga.

\section{INTRODUCTION}

Les métaux sont naturellement présents dans la croute terrestre. Ce sont des éléments métalliques naturels, de densité supérieure à $5 \mathrm{~g} / \mathrm{cm}^{3}$ et tout métal ayant un numéro atomique élevé, en général supérieur à celui du sodium ( $Z=11)$. Ils sont présents dans tous les compartiments de l'environnement, mais généralement à l'état de traces. Les éléments naturellement présents dans les sols dont certains sont indispensables aux plantes, font partie des oligo-éléments et des éléments Traces. On utilise également l'expression métaux lourds, qui correspond à une définition physique (masse volumique supérieure à $5 \mathrm{~g} / \mathrm{cm} 3$ ) ou bien oligoéléments. Les éléments traces les plus connus pour leur dangerosité sont le plomb $(\mathrm{Pb})$, le mercure $(\mathrm{Hg})$, le cadmium (Cd), le chrome ( $\mathrm{Cr}$ ), le cuivre (Cu), le nickel (Ni), le zinc (Zn). II faut ajouter à cette liste l'arsenic (As) et le sélénium (Se), qui ne sont que des Eléments Traces et pas des métaux (Haydon et Cobett, 2007 ; Part et al. 2012). Les éléments traces, avec toute leur importance, peuvent s'accumuler et devenir toxique dans l'environnement par les activités humaines telles que l'exploitation des mines. Cette toxicité peut avoir comme conséquence une accumulation de l'élément dans la chaine alimentaire. Ce qui pourrait sans doute provoquer des dysfonctionnements dans les organismes des animaux et des humains (Baise et Paquereau, 1997 ; Li et al. 2012). L'intérêt des plantes vivrières s'est accru à la suite des études épidémiologiques mettant en relation les habitudes alimentaires et la prévalence de certaines maladies (cancers, obésité, maladies cardiovasculaires) (Diallo et al. 2013). En plus de leur importance nutritionnelle, les plantes vivrières présentent un intérêt économique et social non négligeable en raison de leur cout relativement bas, de la facilité et de la rapidité de leur préparation (Avallone et al. 2007 ; Dansi et al. 2008 ; Vadouhe et al. 2012). Plusieurs de ces plantes vivrières peuvent être exposées aux métaux lourds. D'une part, par voie par aérienne, les métaux lourds sont présents où déposés à la surface des organes (feuilles, tiges) et ils pénètrent dans les stomates sous forme de particules, de composés gazeux ( $\mathrm{Hg}$, As, etc.), ou dissous dans les eaux de pluie ou d'irrigation. Seule une partie de ces dépôts est enlevée par lavage. Les métaux lourds peuvent aussi être absorbés par les racines dans la solution du sol. L'importance relative de ces deux voies d'exposition dépend des métaux lourds, des plantes, de la proximité d'une source de contamination (émissions industrielles ou urbaines, routes) (Baise et Paquereau, 1997 ; Li et al. 2012 ; 


\section{Kalonda et al. J. Appl. Biosci. 2015 Profil des métaux lourds contenus dans les plantes vivrières}

consommées couramment dans quelques zones minières de la province du Katanga.

Mihali et al. 2012). Ce travail s'était proposé de déterminer le profil de différents métaux lourds contenus dans Manihot esculenta (Sombe); Amaranthus Sativa (Lengalenga); et Psidium

\section{METHODES}

Cette étude a porté sur l'analyse des échantillons des feuilles de Manihot esculenta, Amaranthus Sativa et $P$ sidium guajava $L$. Ces plantes ont été récoltées aux alentours des habitations de trois sites miniers dans la province du Katanga, en République Démocratique du Congo : Hewa Bora, Kansonga et Shinkolobwe. Pour la même plante, il y a eu au total trois échantillons prélevés successivement dans les trois sites miniers. Les analyses ont été effectuées par ICP-MS, permettant de doser la quasi-totalité des éléments simultanément à l'état de traces et ultra-traces (Baise et Paquereau, 1997). Pour arriver à l'obtention des résultats, le centre d'expertise

\section{RESULTATS}

Manihot Esculenta : Pour le cobalt, la teneur la plus élevée est de $54,87 \mathrm{mg} / \mathrm{kg}$ (site Kansonga) tandis que la teneur la plus basse est de $11,79 \mathrm{mg} / \mathrm{kg}$ (site Hewabora). Pour le fer, la teneur la plus élevée est de 257,8 $\mathrm{mg} / \mathrm{kg}$ (site Kansonga) tandis que la teneur la plus basse est de 222,1 mg/kg (site Shinkolobwe). Toutefois, le nickel n'a pas été décelé dans les échantillons de Manihot esculenta (sombe) de tous les sites de notre étude. guajava L. (Mapela) plantes couramment consommées dans les zones aux alentours des sites miniers de la province du Katanga, en République Démocratique du Congo.

d'évaluation et de certification des substances minérales précieuses et semi-précieuse (CEEC), laboratoire agréé par l'état congolais, a été le cadre idéal pour les analyses. Après séchage, broyage et réduction en fine poudre des feuilles de ces plantes; elles avaient subies une minéralisation. Elles avaient été analysées en tenant compte des normes d'analyses des plantes (normes USF EPA 3051) et celles d'analyses des métaux lourds (Normes US EPA CLP ILM 053 D et SW-84 66020/6020 A) (Grobelak et Napora, 2015; Park et al. 2012; Koumolou et al. 2012).

Psidium guajava : Pour le nickel, la teneur la plus élevée est de $19,19 \mathrm{mg} / \mathrm{kg}$ (site Shinkolobwe) tandis que la teneur est nulle dans les échantillons de Hewa-bora et de Kansonga. Pour le cobalt, la teneur la plus élevée est de $76,84 \mathrm{mg} / \mathrm{kg}$ (site Shinkolobwe) tandis que la teneur la plus basse est de 4,653mg/kg (site Hewa-bora). Pour le fer, la teneur la plus élevée est de $900,2 \mathrm{mg} / \mathrm{kg}$ (site Shinkolobwe) tandis que la teneur la plus basse est de $218,1 \mathrm{mg} / \mathrm{kg}$ (site Hewa-bora).

Tableau 1. Résultats du dosage par ICP-MS des métaux dans les échantillons des plantes.

\begin{tabular}{|c|c|c|c|c|c|c|c|}
\hline $\mathbf{N}^{\circ}$ & Plante & $\begin{array}{l}24 \mathrm{Mg} \\
\mathrm{mg} / \mathrm{kg}\end{array}$ & $\begin{array}{l}27 \mathrm{Al} \\
\mathrm{mg} / \mathrm{kg}\end{array}$ & $\begin{array}{l}52 \mathrm{Cr} \\
\mathrm{mg} / \mathrm{kg}\end{array}$ & $\begin{array}{l}54 \mathrm{Fe} \\
\mathrm{mg} / \mathrm{kg}\end{array}$ & $\begin{array}{l}59 \mathrm{Co} \\
\mathrm{mg} / \mathrm{kg}\end{array}$ & $\begin{array}{l}60 \mathrm{Ni} \\
\mathrm{mg} / \mathrm{kg} \\
\end{array}$ \\
\hline 1 & $\begin{array}{l}\text { Manihot E. } \\
\text { (Hewa Bora) }\end{array}$ & 4045 & 6832 & 3,357 & 256,5 & 11,79 & 0 \\
\hline 2 & $\begin{array}{l}\text { Manihot E. } \\
\text { (Kansonga) }\end{array}$ & 5867 & 7835 & 3,771 & 257,8 & 54,87 & 0 \\
\hline 3 & $\begin{array}{l}\text { Manihot E. } \\
\text { (Shinkolobwe) }\end{array}$ & 4796 & 6719 & 6,604 & 222,1 & 33,71 & 0 \\
\hline 4 & $\begin{array}{l}\text { Psidium G. } \\
\text { (Hewa Bora) }\end{array}$ & 6482 & 6580 & 2,62 & 218,1 & 4,653 & 0 \\
\hline 5 & $\begin{array}{l}\text { Psidium G. } \\
\text { (Kansonga) }\end{array}$ & 3958 & 6725 & 3,363 & 253,7 & 24,08 & 0 \\
\hline 6 & $\begin{array}{l}\text { Psidium G. } \\
\text { (Shinkolobwe) }\end{array}$ & 6929 & 7524 & 4,455 & 900,2 & 76,84 & 19,19 \\
\hline 7 & Amaranthus S. (Hewa Bora) & 23000 & 7888 & 5,666 & 113,4 & 11,71 & 0 \\
\hline 8 & Amaranthus S. (Kansonga) & 20290 & 8919 & 18,51 & 1642 & 116,2 & 5,863 \\
\hline 9 & Amaranthus S. (Shinkolobwe) & 24250 & 5173 & 6,478 & 112,7 & 77,15 & 5,484 \\
\hline
\end{tabular}


Amaranthus Sativa : Pour le nickel, la teneur la plus élevée est de $5,863 \mathrm{mg} / \mathrm{kg}$ (site Kansonga) tandis que la teneur est nulle à Hewa-bora. Pour le cobalt, la teneur la plus élevée est de $116,2 \mathrm{mg} / \mathrm{kg}$ (site Kansonga) tandis que la teneur la plus basse est de $11,71 \mathrm{mg} / \mathrm{kg}$ (site Hewa-bora). Pour le fer, la teneur la plus élevée est de $1642 \mathrm{mg} / \mathrm{kg}$ (site Kansonga) tandis que la teneur la plus basse est de 112,7 mg/kg (site Shinkolobwe). Tous les autres détails des résultats sont inscrits ci dessous (tableau 1).

Manihot Esculenta : Pour l'uranium, la teneur la plus élevée est de $0,22 \mathrm{mg} / \mathrm{kg}$ (site Kansonga) tandis que la teneur la plus basse est de 0,169 mg/kg (site Hewabora). Pour le plomb, la teneur la plus élevée est de 5,21 $\mathrm{mg} / \mathrm{kg}$ (site Kansonga) tandis que la teneur la plus basse est de $3,331 \mathrm{mg} / \mathrm{kg}$ (site Hewa-bora). Pour le zinc, la teneur la plus élevée est de $717 \mathrm{mg} / \mathrm{kg}$ (site Kansonga) tandis que la teneur la basse est de $418,9 \mathrm{mg} / \mathrm{kg}$ (site Shinkolobwe). Pour le cuivre, la teneur la plus élevée est de $67,24 \mathrm{mg} / \mathrm{kg}$ (site Kansogna) tandis que la teneur la plus basse est de 30,28 mg/kg (site Hewa-bora).

Psidium guajava: Pour l'uranium, la teneur la plus élevée est de $0,452 \mathrm{mg} / \mathrm{kg}$ (site Shinkolobwe) tandis que la teneur la plus basse est de $0,222 \mathrm{mg} / \mathrm{kg}$ (site Hewabora). Pour le plomb, la teneur la plus élevé est de 5,797 $\mathrm{mg} / \mathrm{kg}$ (site Shinkolobwe) tandis que la teneur la plus basse est de 4,089 mg/kg (site Kansonga). Pour le cadmium, la teneur la plus élevée est de $0,139 \mathrm{mg} / \mathrm{kg}$ (site Shinkolobwe) tandis que la teneur la plus basse est de $0,059 \mathrm{mg} / \mathrm{kg}$ (site Hewa-bora).

Pour le zinc, la teneur la plus élevée est de $393,2 \mathrm{mg} / \mathrm{kg}$ (site Shinkolobwe) tandis que la teneur la plus basse est de $279,3 \mathrm{mg} / \mathrm{kg}$ (site Kansonga). Pour le cuivre la teneur la plus élevée est de $200,1 \mathrm{mg} / \mathrm{kg}$ (site Shinkolobwe) tandis que la teneur la plus basse est de $50,39 \mathrm{mg} / \mathrm{kg}$ (site Kansonga).

Amaranthus Sativa : Pour l'uranium, la teneur la plus élevée est de $0,534 \mathrm{mg} / \mathrm{kg}$ (site Kansonga) tandis que la teneur la plus basse est de 0,302 mg/kg (site Hewabora). Pour le plomb, la teneur la plus élevée est de $10,25 \mathrm{mg} / \mathrm{kg}$ (site Kansonga) tandis que la teneur la plus basse est de $5,352 \mathrm{mg} / \mathrm{kg}$ (site Shinkolobwe). Pour le cadmium, la teneur la plus élevée est de $7,843 \mathrm{mg} / \mathrm{kg}$ (site Shinkolobwe) tandis que la teneur la plus basse est de $2,024 \mathrm{mg} / \mathrm{kg}$ (site Hewa-bora). Pour le zinc, la teneur la plus élevée est de $497,1 \mathrm{mg} / \mathrm{kg}$ (site Kansonga) tandis que la teneur la plus basse est de $370,5 \mathrm{mg} / \mathrm{kg}$ (site Hewa-bora). Pour le cuivre la teneur la plus élevée est de $516,2 \mathrm{mg} / \mathrm{kg}$ (site Shinkolobwe) tandis que la teneur la plus basse est de $45,69 \mathrm{mg} / \mathrm{kg}$ (Site Hewa-bora). Tous les autres détails des résultats sont inscrits ci dessous (tableau 2).

Tableau 2. Résultats du dosage par ICP-MS des métaux dans les échantillons des plantes.

\begin{tabular}{|c|c|c|c|c|c|c|c|}
\hline $\mathrm{N}^{\circ}$ & Plante & $\begin{array}{l}65 \mathrm{Cu} \\
\mathrm{mg} / \mathrm{kg}\end{array}$ & $\begin{array}{l}66 \mathrm{Zn} \\
\mathrm{mg} / \mathrm{kg}\end{array}$ & $\begin{array}{l}114 \mathrm{Cd} \\
\mathrm{mg} / \mathrm{kg}\end{array}$ & $\begin{array}{l}111 \mathrm{Cd} \\
\mathrm{mg} / \mathrm{kg}\end{array}$ & $\begin{array}{l}208 \mathrm{~Pb} \\
\mathrm{mg} / \mathrm{kg}\end{array}$ & $\begin{array}{l}238 \mathrm{U} \\
\mathrm{mg} / \mathrm{kg}\end{array}$ \\
\hline$\overline{11}$ & $\begin{array}{l}\text { Manihot E. } \\
\text { (Hewa Bora) }\end{array}$ & 30,28 & 428,6 & 0,102 & 0,413 & 3,67 & 0,169 \\
\hline 2 & $\begin{array}{l}\text { Manihot E. } \\
\text { (Kansonga) }\end{array}$ & 67,24 & 717 & 1,621 & 2,025 & 5,21 & 0,22 \\
\hline 3 & $\begin{array}{l}\text { Manihot E. } \\
\text { (Shinkolobwe) }\end{array}$ & 58,78 & 418,9 & 0,782 & 1,36 & 3,331 & 0,219 \\
\hline 4 & $\begin{array}{l}\text { Psidium G. } \\
\text { (Hewa Bora) }\end{array}$ & 58,11 & 290,9 & 0,059 & 0,631 & 5,336 & 0,222 \\
\hline 5 & $\begin{array}{l}\text { Psidium G. } \\
\text { (Kansonga) }\end{array}$ & 50,39 & 279,3 & 0,067 & 0,603 & 4,089 & 0,31 \\
\hline 6 & $\begin{array}{l}\text { Psidium G. } \\
\text { (Shinkolobwe) }\end{array}$ & 200,1 & 393,2 & 0,139 & 0,89 & 5,797 & 0,452 \\
\hline 7 & Amaranthus S. (Hewa Bora) & 45,69 & 370,5 & 1,295 & 2,024 & 7,547 & 0,302 \\
\hline 8 & Amaranthus S. (Kansonga) & 217,7 & 497,1 & 1,981 & 2,596 & 10,25 & 0,534 \\
\hline 9 & Amaranthus S. (Shinkolobwe) & 516,2 & 411,3 & 7,717 & 7,843 & 5,352 & 0,473 \\
\hline
\end{tabular}




\section{Kalonda et al. J. Appl. Biosci. 2015 Profil des métaux lourds contenus dans les plantes vivrières}

consommées couramment dans quelques zones minières de la province du Katanga.

\section{DISCUSSION}

Plusieurs observations en rapport avec la présence des métaux lourds dans les plantes découlent des résultats des analyses de nos échantillons. Les zones industrielles sont caractérisées par des processus de dégradation des sols qui sont liés principalement au dépôt de métaux lourds (Mortvet, 1996; Baise et Paquereau, 1997 ; Grobelak et Napora, 2015). Les zones contaminées par des métaux lourds sont une grave source de risque en raison des émissions des polluants secondaires et de la lixiviation des métaux. Considérant les taux de Nickel (Ni), la valeur normale étant fixée dans l'intervalle allant $1-50 \mu \mathrm{g} / \mathrm{g}$ soit : $1000-50000 \mu \mathrm{g} / \mathrm{Kg}$ ou $1-50 \mathrm{mg} / \mathrm{Kg}$; nos résultats montrent que les valeurs trouvées sont dans la limite, excluant ainsi tout danger possible pour les consommateurs (Mihali et al. 2012). Par rapport au Zinc (Zn), la valeur limite étant fixée entre $1-100 \mathrm{mg} / \mathrm{Kg}$, les résultats trouvés montrent une élévation dans tous les échantillons dont les taux vont de $717 \mathrm{mg} / \mathrm{Kg}$ à 290,9 $\mathrm{mg} / \mathrm{Kg}$. Ces taux sont alarmants, car constituent un danger important pour les habitants qui en consomment (Koumolou et al. 2012). Pour le Cuivre (Cu), la valeur limite acceptable est fixée à $100 \mathrm{mg} / \mathrm{Kg}$; les résultats montrent que dans certains échantillons les valeurs étaient supérieures, respectivement $516,2 \mathrm{mg} / \mathrm{Kg}, 217,7$ $\mathrm{mg} / \mathrm{Kg}$ et $200,1 \mathrm{mg} / \mathrm{kg}$, toutefois les autres valeurs restantes étaient dans la limite normale. Nous pensons que certaines situations aboutissent à une contamination plus marquée du sol et à un danger plus grand de contamination des végétaux cultivés ; c'est notamment le cas des parcelles de cultures intensives spécialisées qui peuvent avoir été contaminées par des produits phytosanitaires contenant du zinc, mercure, plomb, arsenic ou cuivre; ou par une fertilisation intense (Cadmium) (Baise et Paquereau, 1997). II nous parait aussi évident que des parcelles à proximité d'usines métallurgiques, d'exploitations minières ou d'installations polluantes sont contaminées par voie atmosphérique ou par la dispersion accidentelle de déchets (Haydon et Cobbett, 2007 ; Park et al. 2012). Par rapport au Cadmium (Cd), la valeur limite est de $0,2 \mathrm{mg} / \mathrm{Kg}$; les résultats ont montré des teneurs supérieures à la limite normale dans certains échantillons : 7,717 mg/Kg, 1,981

\section{CONCLUSION}

La majorité des plantes vivrières présentent une contamination en métaux lourds préoccupante. Les métaux lourds étaient présents dans les plantes récoltées aux alentours des zones minières à différentes concentrations. La plupart des échantillons avait présenté $\mathrm{mg} / \mathrm{Kg}, 1,621 \mathrm{mg} / \mathrm{Kg}, 1,245 \mathrm{mg} / \mathrm{kg}$ et $0,782 \mathrm{mg} / \mathrm{Kg}$. Les recherché faites en France, avaient montré qu'une contamination diffuse des sols en éléments traces par les activités humaines demeure faible. Par ailleurs l'augmentation du stock de $\mathrm{Cd}$, $\mathrm{Zn}$ et Ni dans l'horizon labouré est à surveiller, car ces métaux passent assez facilement dans la chaine alimentaire (Baise et Paquereau, 1997). Concernant le Plomb (Pb), les valeurs normales sont reparties selon les espèces soit 0,10 $\mathrm{mg} / \mathrm{Kg}$ pour les plantes non légumineuse comme le cas du goyavier et $0,30 \mathrm{mg} / \mathrm{Kg}$ pour les légumineuses (Feuilles de manioc et Amarante). Les résultats trouvés sont de loin supérieurs aux normes ; alors que la nature toxique du plomb est connue depuis longtemps, ainsi que les dangers lies à la contamination des aliments par ce métal (Mourato et al. 2015). En considérant l'Uranium (U), la valeur limite est de $0,36 \mathrm{mg} / \mathrm{Kg}$. Certains échantillons ont montrés les valeurs supérieures à la limité normale. Les investigations de Mordtvet (1996) avaient démontrées que du fait que l'uranium était présent dans tous les sols sous forme partiellement disponible, il était plus ou moins normal de le rencontré dans toutes les plantes. Ainsi, estimons nous que cette pensée corrobore nos recherches. Toutefois, pour Mordtvet (1996), dans les zones très uranifères, on peut observer des modifications de pigmentations chez certaines espèces végétales et des bouleversements dans leur croissance. Considérant tous les éléments ci haut analysés, nous pensons aussi qu'une attention particulière devrait être mise aussi sur les jardins familiaux à proximité d'agglomérations ou d'un site industriel émetteur ; car plus ils sont anciens et inclus dans l'agglomération, plus leur sur-contamination est fréquente par rapport aux parcelles agricoles ( $\mathrm{Li}$ et al. 2012; Koumolou et al. 2012). Les activités d'assainissement qui sont appliqués à grande échelle dans des zones contaminées par des métaux lourds devraient être principalement axées sur la réduction du dégré de mobilité des métaux dans le profil du sol et la biodisponibilité des métaux à des niveaux qui ne sont pas phytotoxiques (Grobelak et Napora, 2015).

des taux en métaux lourds relativement élevés ; fait lier probablement à la transformation industrielle, l'exploitation minière et les pratiques agricoles. Cette contamination pourrait être à la base de beaucoup des maladies chez les humains consommateurs, mais aussi 


\section{Kalonda et al. J. Appl. Biosci. 2015 Profil des métaux lourds contenus dans les plantes vivrières consommées couramment dans quelques zones minières de la province du Katanga.}

de beaucoup des dommages dans la production agricole comme dans l'élevage.

RECOMMANDATIONS: Les ministères de l'environnement, de l'infrastructure, ensemble avec celui de la santé devraient veiller que les habitations des humains avec leurs cultures agricoles soient loin des sites miniers. Car il y a une possibilité de contamination des sols pour les cités environnants les zones minières ; et cette situation pourrait être la cause de plusieurs pathologies connues comme pour les non connues.

Conflits d'intérêt : Aucun.

Contributions des auteurs : Tous les auteurs ont contribué à la réalisation de ce travail ; ils ont lu et approuvé la version finale du manuscrit.

\section{REFERENCES BIBLIOGRAPHIQUES}

Avallone S., Brault S., Mouquet C., Trèche S. (2007). Home-processing of the dishes constituting the main sources of micronutrients in the diet of preschool children in rural Burkina Faso. Int. J. Food. Sci. Nutr. $58: 108-115$.

Baise D. et Paquereau H. (1997). Teneurs totales en élements traces dans les sols agricoles de Seine-et-Marne. Etude et Gestion des sols. 4(2) :77-94.

Dansi A., Adjatin A., Adoukonou-Sagbadja H., Falade V., Yedomonhan H., Odou D., Dossou B. (2008). Traditional leafy vegetables and their use in the Benin Republic. Genet. Resour. Crop EV. $55: 1239$ - 1256.

Diallo Y., Gueye M.T., Sakho M., Darboux P.G., Kane A., Barthelemy J.P., Lognay G. (2013). Importance nutritionnelle du manioc et perspectives pour l'alimentation de base au Sénegal (synthèse bibliographique). Biotechnol. Agron. Soc. Environ. 17(4):634-643.

Grobelak A., Napora A. (2015). The Chemophytostabilisation Process of Heavy Metal Polluted Soil. PLoS One. 10(6):e0129538.

Haydon M.J., Cobbett C.S. (2007). Transporters of ligands for essential metal ions in plants. New Phytol. 174:499-506.

Koumolou L., Edorh A.P., Agbandji L., Hounkpatin S.A., Elegbede B. (2012). Threat of the health quality of garden produces linked to pollution by toxic metals on some gardening sites of Benin. Am. J. Environ. Sci. 8(3): $248-52$.

Li Q.S., Chen Y., Fu H.B., Cui Z.H., Shi L., Wang L., Liu Z.F. (2012). Health risk of heavy metals in food crops growm on reclaimed tidal flat soil in the pearl River Estuary, China. J Hazard Mater. 227-228(7):148-54.

Mihali C., Michnea A., Oprea G., Gogoasa I., Pop C., Senila M., Grigor L. (2012). Trace element transfer from soil to vegetables around the lead smelter in Baia Mare, NW Romania. J Food Agr Environ. 10(1) :828 - 34 .

Mortvet J.J. (1996). Plant and soil relationships of uranium and thorium decay series radionuclides. A review. J Environ Qual. 23:643 - 650.

Mourato M.P., Moreira I.N., Leitão I., Pinto F.R., Sales J.R., and Martins L.L.(2015). Effect of Heavy Metals in Plants of the Genus Brassica. Int J Mol Sci. 16(8): 17975-17998.

Park J., Kim J.-Y., Kim K.-W. (2012).Phytoremediation of soil contaminated with heavy metals using Brassica napus. Geosyst. Eng. 15:9-17.

Vadouhe S., Dovoedo A., Anihouvi V.B., Tossou R.C., Soumanou M.M. (2012). Influence du mode de cuisson sur la valeur nutritionnelle de Solanum macrocarpum, Amaranthus hybridus et Ocimum gratissimum, trois légumes-feuilles traditionnels acclimatés au Bénin. Int. J. Biol. Chem. Sci. 6(5): :1926 - 1937. 